\title{
Impaired Error Awareness and Anterior Cingulate Cortex Hypoactivity in Chronic Cannabis Users
}

\author{
Robert Hester*,', Liam Nestor ${ }^{2}$ and Hugh Garavan ${ }^{2}$ \\ 'Department of Psychology, University of Melbourne, Melbourne, Victoria, Australia; ${ }^{2}$ School of Psychology and Trinity College Institute of \\ Neuroscience, Trinity College Dublin, Dublin, Ireland
}

\begin{abstract}
Drug abuse and other psychiatric conditions (eg, schizophrenia) have been associated with a diminished neural response to errors, particularly in the anterior cingulate cortex (ACC) thought critical to error processing. A diminished capacity for detecting errors has been linked to clinical symptoms including the loss of insight, delusions, and perseverative behavior. A total of I 6 active chronic cannabis users and 16 control participants were administered a Go/No-go response inhibition task during event-related fMRI data collection. The task provides measures of inhibitory control and error awareness. Cannabis users' inhibitory control performance was equivalent to that of the control group, but the former showed a significant deficit in awareness of commission errors. Cannabis users showed a diminished capacity for monitoring their behavior that was associated with hypoactivity in the ACC and right insula. In addition, increased levels of hypoactivity in both the ACC and right insula regions were significantly correlated with error-awareness rates in the cannabis group (but not controls). These difficulties are consistent with earlier reports of hypoactivity in the neural systems underlying cognitive control and the monitoring of interoceptive awareness in chronic drug users, and highlight the potential relationship between cognitive dysfunction and behavioral deficits that have the potential to contribute to the maintenance of drug abuse.

Neuropsychopharmacology (2009) 34, 2450-2458; doi:I 0.1038/npp.2009.67; published online 24 June 2009
\end{abstract}

Keywords: performance monitoring; error-related; drug addiction; marijuana; insula; cognitive control

\section{INTRODUCTION}

Healthy adults are very good at detecting cognitive failures, whereas a common feature of many psychiatric and neurological conditions is a diminished capacity for performance monitoring (Ullsperger, 2006). Deficits in error detection have also been found to relate to clinical symptoms, including the debilitating symptoms of loss of insight (Lysaker et al, 1998), perseverative behavior (Frith, 1987) and delusions of alien control in schizophrenia (Frith and Done, 1989), and poor clinical outcomes (eg, inability to maintain independent living (Seltzer et al, 1997)).

Consistent with behavioral symptoms of impaired performance monitoring, past research has repeatedly identified diminished error-related anterior cingulate cortex (ACC) activity in a range of clinical conditions, including schizophrenia (Alain et al, 2002; Carter et al, 2001; Turken et al, 2003), major depression (Steele et al, 2007), and drug addiction (Forman et al, 2004; Kaufman et al, 2003). Performance-monitoring deficits in drug abusing popula-

*Correspondence: Dr Robert Hester, Department of Psychology, University of Melbourne, Melbourne, Victoria 3010, Australia,

Tel.: + 613 83440222, Fax: +61393476618,

E-mail: hestern@unimelb.edu.au

Received 27 January 2009; revised 27 April 2009; accepted 19 May 2009 tions are of interest because they relate not only to features such as loss of insight, but also to dysfunction in the cognitive control system (Ridderinkhof et al, 2004). Cognitive control processes are fundamental to the ability to inhibit the immediate pursuit of pleasurable stimuli, and for the development of adaptive patterns of behavior - both key factors in drug abuse (Kalivas and Volkow, 2005). Earlier research has not examined performance monitoring in chronic cannabis users, although it is of interest due to cannabis's links with executive dysfunction and the emergence of psychotic symptoms.

Current evidence suggests that the neural response to errors involves a network of regions (Ridderinkhof et al, 2004), which consistently involves the dorsal ACC. Studies specifically examining the neural correlates of error awareness have implicated much of the same error-related cortical network (Hester et al, 2005; Klein et al, 2007; Nieuwenhuis et al, 2001; O'Connell et al, 2007), with awareness most strongly associated with activity in the insula (anterior inferior portion), right dorsolateral prefrontal, and bilateral parietal regions. Each of these studies found that error-related ACC activity was present for both aware and unaware errors, but did not differentiate between them, suggesting that ACC activity may be necessary, but not sufficient, for error awareness.

Given the relationship between error-related ACC activity and error awareness in healthy adults, it remains unclear 
whether the poor error awareness found in psychiatric groups is related to their diminished error-related ACC activity. Similarly, cortical regions that seem critical to error awareness, such as the insula cortex, have been associated with insight problems in drug abuse (Paulus, 2007). The aim of this study was to examine the neural correlates of error awareness in chronic cannabis users, using a task we have earlier verified as sensitive to the neural mechanisms underlying error awareness (Hester et al, 2005) and awareness impairments in drug abuse (Hester et al, 2007). Earlier research with chronic cannabis users has identified diminished ACC activity during executive control tasks (Bolla et al, 2004; Eldreth et al, 2004; Gruber and YurgelunTodd, 2005), but no study to date has examined performance monitoring.

\section{MATERIALS AND METHODS}

\section{Subjects}

A total of 16 active cannabis users ( 1 female, mean age $=24.7$, range $=18-40)$ and 16 matched control participants ( 1 female, mean age 25.3, range: $20-36$ ) were recruited from University campuses within Dublin City, Ireland, through leaflet advertising. After complete description of the study to the subjects, written informed consent was obtained. Groups were also matched for educational attainment (control: 17.8 years, cannabis: 16.2, $F(1,31)=$ 2.71, $p=0.11$ ) and National Adult Reading Test (NART) estimated IQ (control: 124.0, cannabis: 123.3, $F(1,31)=0.26$, $p=0.61$ ). A semi-structured interview was used to screen participants for past or present history of psychiatric or neurological illness. All participants completed inventories of drug use (questionnaire taken from the Addiction Severity Index Lite-CF) to screen for past or concurrent abuse of other substances. Prospective participants from either sample were additionally considered ineligible if they reported concurrent or past dependence on other drugs (including nicotine and alcohol). Information concerning alcohol and cannabis use in each participant was indexed in number of years (lifetime) and occasions of recent use (last 30 days) and is presented in Table 1. The groups did not differ on any measure of drug use other than those relating to cannabis.

Participants in the cannabis group were required to have regularly consumed cannabis (5-7 days/week) for the earlier 2 years and to have smoked a minimum of 500 joints in their lifetime to be eligible for the study. All cannabis users provided a positive urine sample for $\Delta^{9}$-tetrahydrocannabinol $\left(\Delta^{9} \mathrm{THC}\right)$ before scanning, with an additional screening for other confounding drug use (Cozart RapiScan, Abingdon, UK) taking place. Control participants were also tested for $\Delta^{9} \mathrm{THC}$ and the above adulterants. Participants providing positive tests for drugs other than cannabis (cannabis group only) or alcohol were excluded, and all participants provided a breath test $0.00 \%$ blood alcohol concentration reading before the beginning of the cognitive testing.

\section{Behavioral Tasks}

Error-awareness task. To examine conscious recognition of errors we administered the error-awareness task (EAT) (see
Table I Mean and SEM for Control and Cannabis Groups on Demographic and Drug use History

\begin{tabular}{lcc}
\hline & $\begin{array}{c}\text { Control } \\
(\mathbf{n}=\mathbf{I 6})\end{array}$ & $\begin{array}{c}\text { Cannabis } \\
(\mathbf{n}=\mathbf{I 6})\end{array}$ \\
\hline Age & $25.2 \pm 1.3$ & $24.6 \pm 1.5$ \\
Years of education & $17.7 \pm 0.7$ & $16.2 \pm 0.7$ \\
Verbal intelligence score (NART) & $124.0 \pm 0.8$ & $123.3 \pm 0.8$ \\
Beck depression inventory II score & $4.0 \pm 0.7$ & $5.5 \pm 1.1$ \\
Females/males & $1 / 15$ & $1 / 15$ \\
& & \\
Years of alcohol use & $8.7 \pm 1.4$ & $8.9 \pm 1.4$ \\
Alcohol use in last month (no. of days) & $6.2 \pm 1.4$ & $9.3 \pm 1.8$ \\
Alcohol use age onset (years) & $16.2 \pm 0.6$ & $15.8 \pm 0.5$ \\
Cannabis use (years) & $0.0 \pm 0.0$ & $8.2 \pm 1.3$ \\
Lifetime joints (number) & $3.0 \pm 0.6$ & $11628.8 \pm 5993.4$ \\
Days of use in last month (number) & $0.0 \pm 0.0$ & $19.2 \pm 2.6$ \\
Joints in last month (number) & $0.0 \pm 0.0$ & $76.3 \pm 17.7$ \\
Cannabis use age onset (years) & $17.0 \pm 0.3$ & $16.4 \pm 0.7$ \\
Cannabis abstinence (h) & & $38.0 \pm 47.7$ \\
Cannabis withdrawal score (out of 32) & & $9.3 \pm 2.2$ \\
Cannabis craving scores (each item out of 21) & & \\
Compulsivity & & $6.0 \pm 1.0$ \\
Emotionality & & $8.4 \pm 1.1$ \\
Expectancy & & $11.3 \pm 1.3$ \\
Purposefulness & & $10.1 \pm 1.3$ \\
\hline
\end{tabular}

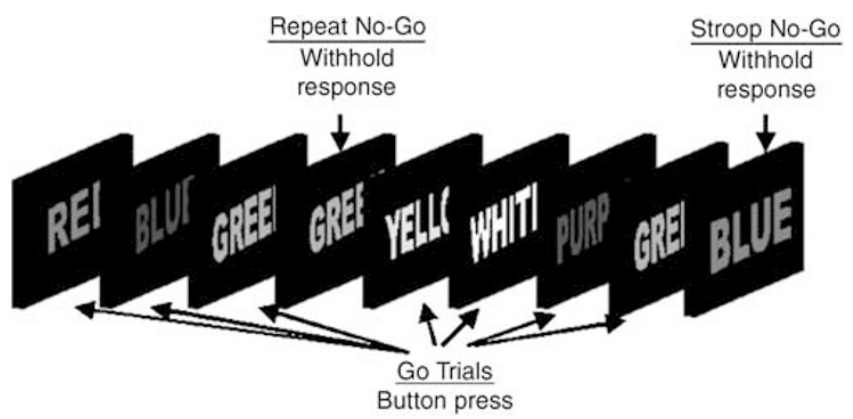

Figure I The error-awareness task. The EAT presents a serial stream of single color words in congruent fonts, with the word presented for $900 \mathrm{~ms}$ followed by a $600 \mathrm{~ms}$ inter-stimulus interval. Participants were trained to respond to each of the words with a single 'Go trial' button press, and withhold this response when either of two different circumstances arose. The first was if the same word was presented on two consecutive trials (Repeat No-go), and the second was if the word and font of the word did not match (Stroop No-go). To indicate 'error awareness' participants were trained to press the go-trial button twice on the trial following any commission errors.

Figure 1) (Hester et al, 2005), a motor Go/No-go responseinhibition task in which subjects make errors of commission of which they are aware (aware errors), or unaware (unaware errors). The EAT presents a serial stream of single color words in congruent fonts, with the word presented for $900 \mathrm{~ms}$ followed by a $600 \mathrm{~ms}$ inter-stimulus interval. Participants were trained to respond to each of the words with a single 'Go trial' button press, and withhold this 
response when either of two different circumstances arose. The first was if the same word was presented on two consecutive trials (Repeat No-go), and the second was if the word and font of the word did not match (Stroop No-go). By having competing types of response inhibition rules, we aimed to vary the strength of stimulus-response relationships, whereby representations of rules competitively suppress one another such that the more prepotent rule would suppress the weaker rule and so produce a significant number of errors, a small proportion of which may go unnoticed because of focusing primarily on the prepotent rule. In particular, we aimed to capitalize on the overlearned human behavior of reading the word, rather than the color of the letters (the Stroop effect), and so predispose participants to monitor for the Repeat, rather than the Stroop, No-go's. To indicate 'error awareness' participants were trained to press the go-trial button twice on the trial following any commission errors.

Before entering the MRI scanner, participants practiced two novel blocks of the task to ensure that they understood the task instructions. Five blocks of 225 trials (200 Go trials, 25 No-go trials) for a total of 1125 trials were administered during MRI data collection, with each block separated by a short break. An equivalent number of Stroop and Repeat No-go trials were administered across the five blocks for a total of 62 repeat and 63 Stroop No-go trials. All aspects of stimulus delivery and response recording were controlled by E-Prime software (version 1.1 Psychology Software Tools, Pittsburgh, PA), running on a laptop PC (Celeron $2 \mathrm{Ghz}, 128 \mathrm{MB}$ Nvidia Video Card), which was interfaced with the MR scanner during acquisition of fMRI data. Stimuli were back projected onto a screen at the head of the scanner bed, with a head-coil mounted mirror enabling participants to view stimuli. Participants responded to each stimulus using their right hand, entering their response on an MR-compatible response box (Fibre-Optic response pads, Current Designs, Philadelphia, PA).

\section{Image Acquisition}

Functional MR images were acquired at Trinity College Institute for Neuroscience, Dublin City, Ireland, using a Philips Intera Achieva 3.0 Tesla MR system (Best, The Netherlands) with a gradient-echo echo-planar imaging (EPI) sequence. The scanner was equipped with a radiofrequency birdcage head coil for signal transmission and reception. Lateral head stabilizers were used to minimize head movement. EPI images were acquired using a gradient-echo pulse sequence and sequential slice acquisition $\left(T_{R}=2000 \mathrm{~ms}\right.$, $\mathrm{T}_{\mathrm{E}}=35 \mathrm{~ms}$, flip angle $=90^{\circ}, 32$ non-contiguous slices of $3.5 \mathrm{~mm}$ thickness, $10 \%$ gap, in-plane resolution of $3.5 \times 3.5$ pixels in a FOV of $224 \mathrm{~mm}$ ). Each functional run began with four volume acquisitions that were later discarded, to allow for steady-state tissue magnetization. Activation data were registered to high-resolution T1-weighted isotropic $\left(0.9 \mathrm{~mm}^{3}\right)$ structural MPRAGE images to localize the pattern of physiological changes associated with the task.

\section{Data analysis}

Behavioral data from each participant were used to categorise the No-go trial events into successful responses (stops), aware errors, and unaware errors. All analyses were conducted using AFNI software (http://afni.nimh.nih.gov/ afni/) (Cox, 1996). After image reconstruction, the timeseries data were motion-corrected using 3D volume registration (least-squares alignment of three translational and three rotational parameters). Activation outside the brain was removed using edge-detection techniques.

Separate hemodynamic response functions (HRFs) at 2-s temporal resolution were calculated using deconvolution techniques for aware errors, unaware errors, and stop events. A non-linear regression program determined the best-fitting gamma-variate function for these HRFs as described earlier (Murphy and Garavan, 2005). The area under the curve of the gamma-variate function was expressed as a percentage of the area under the baseline. The baseline in this design is an implicit one and is indicative of task-related go-trial processing that remains after the variance related to the other types of events have been removed.

The percentage area (event-related activation) map voxels were re-sampled at $1 \mathrm{~mm}^{3}$ resolution, then spatially normalized to standard MNI space (MNI 152 template), and spatially blurred with a $3 \mathrm{~mm}$ isotropic rms Gaussian kernel. Group activation maps for event-type (aware errors, unaware errors, and stops) were determined with onesample $t$-tests against the null hypothesis of zero eventrelated activation changes (ie, no change relative to baseline). Significant voxels passed a voxelwise statistical threshold $(t=4.31, p \leqslant 0.001)$ and were required to be the part of a larger $142 \mu \mathrm{l}$ cluster of contiguous significant voxels. By using a combination of probability thresholding and cluster thresholding, the aim is to maximize the power of the statistical test while holding the likelihood of falsepositives to a minimum. To determine the cluster threshold, we use a program called Alphasim (http://afni.nimh.nih.gov/pub/dist/doc/program_help/AlphaSim.html). We provide the program with the number of voxels in the group map, the spatial correlation of voxels (must be contiguous on three sides), and the voxelwise threshold (in this study, $p=0.001)$. The program then runs a series of Monte Carlo simulations (1000 iterations for our study) to determine the frequency of clusters of varying sizes produced by chance. From this frequency distribution, we then select the cluster size ( $142 \mu$ l given our parameters) that occurs $<1 \%$ of the time by chance, to give a threshold of $p=0.01$ (corrected).

The comparison of interest was between aware and unaware errors. The activation clusters from whole-brain analyses of both aware and unaware errors were used to create an OR map for the purposes of a region of interest (ROI) analysis. An OR map includes the voxels of activation indicated as significant from either of the constituent maps. The mean activation for clusters in the combined map was then calculated for the purposes of an ROI analysis, deriving mean activation levels for aware and unaware errors that were compared using repeated measures $t$-tests, corrected through a modified Bonferroni's procedure for multiple comparisons (Keppel, 1991).

The approach of combining aware and unaware error maps was taken because of the relatively small number of unaware error events (on average, 32 aware and 13 unaware errors per subject). Earlier use of this approach with results from this task (Hester et al, 2005) has shown that the 
analysis is not biased toward activity from aware errors. Although the number of events has been shown to influence the spatial extent of activation (Saad et al, 2003), it does not affect the level of activation in a functionally defined ROI-type analysis (Murphy and Garavan, 2005) as used here.

To confirm that activation seen during aware errors did not represent the changed response demands (ie, altering the response to indicate awareness), we have administered earlier an Oddball condition during MRI data collection to identify activations associated with the changed response demands of the aware errors. This condition replicated the stimuli and timing from the EAT task. Oddball events therefore represented similar response and decision requirements to aware errors, without the subject making an error. The Oddball condition indicated significant activation (greater than zero) in only one of the aware ROIs (left middle temporal gyrus, Brodmann's area (BA) 21 ), confirming that the differences between aware and unaware errors did not result from this requirement.

\section{RESULTS}

\section{Behavioral Results}

Performance indices for both control and cannabis participants are presented in Table 2. Control participants' inhibitory control, as measured by No-go accuracy, was not significantly different to cannabis participants, $F(1,31)=$ $0.45, p=0.83$. Control participants were aware of over $91 \%$ of their No-go errors, a significantly higher proportion in comparison to $77 \%$ for cannabis users, $F(1,31)=5.27$, $p=0.03$. Control participants' Go trial response speed was not significantly different to the cannabis group. Go RTs were significantly slower than aware error RTs for both groups, but no difference was found for unaware errors.

In summary, the cannabis group displayed equivalent inhibitory control performance, but significantly poor error awareness.

Table 2 Mean Accuracy, Reaction Time, and Standard Deviation Scores for Cannabis $(n=16)$ and Control $(n=16)$ Groups on the Error-Awareness Task

\begin{tabular}{|c|c|c|c|c|c|}
\hline \multirow[t]{2}{*}{ Category } & \multicolumn{2}{|c|}{ Cannabis } & \multicolumn{2}{|c|}{ Control } & \multirow[t]{2}{*}{$P$-values } \\
\hline & $\mathbf{M}$ & SD & $\mathbf{M}$ & SD & \\
\hline No-go accuracy (\% correct) & 53.2 & 22.4 & 51.6 & 19.2 & \\
\hline Repeat No-go accuracy & 63.8 & 21.1 & 61.3 & |8.1 & \\
\hline Color No-go accuracy & 42.9 & 26.5 & 42.2 & 21.4 & \\
\hline Error awareness ( $\%$ of aware errors) & 77.5 & 24.4 & 91.9 & 6.6 & * \\
\hline Repeat error awareness & 75.2 & 28.1 & 90.0 & 12.2 & * \\
\hline Color error awareness & 79.5 & 23.1 & 93.7 & 5.6 & * \\
\hline Go RT (ms) & 515.4 & 98.1 & 531.4 & 81.8 & \\
\hline \multicolumn{6}{|l|}{ Error RT (ms) } \\
\hline Aware error RT (ms) & 437.7 & 136.4 & 443.8 & 123.6 & \\
\hline Unaware error RT (ms) & 513.9 & |69.1 & 521.2 & 160.9 & \\
\hline
\end{tabular}

*Significant difference between cannabis and control groups $(p<0.05)$.
Relationship between cannabis-use behavior and cognitive task performance. No-go accuracy and error-awareness indices from the EAT task were examined for relationship to self-report measures of cannabis use in the cannabis group. No-go accuracy did not significantly correlate with any of the self-report measures. However, approaching significance was the association with the reported age of onset for cannabis use $(r=0.42, p=0.10)$, whereby earlier onset of cannabis use was associated with poor inhibitory control. Significant relationships were identified between indices derived from the Marijuana Craving questionnaire and individual differences in error awareness, specifically emotionality $(r=0.54, p=0.03)$ and purposefulness $(r=0.51, p=0.04)$. These constructs characterized the questions that are related to the use of cannabis in anticipation of relief from withdrawal or negative mood (emotionality), and the intention and planning to use cannabis because of its earlier rewarding/positive mood inducing qualities (purposefulness) (Heishman et al, 2001). The positive relationship observed indicates that heightened states of 'emotional craving' were associated with poor error awareness.

\section{Imaging Data}

Errors. An analysis combining all participants showed that No-go errors were associated with significant activity in the posterior medial frontal cortex (see Figure 2). The centreof-mass for this cluster of activity was located in the right dorsal ACC (MNI co-ordinates: $x=2 ; y=21 ; z=36$ ), which falls within the rostral cingulate zone highlighted by Ridderinkhof et al (Ridderinkhof et al, 2004) review of performance monitoring. Within this functionally defined ROI, aware errors were associated with significantly higher levels of BOLD activity compared with unaware errors. Activity in several other regions also differentiated aware from unaware errors (see Table 3 and Figure 2), including right insula, bilateral inferior parietal, right middle frontal (BA 6), right putamen, right middle occipital, right cerebellum, and left middle frontal cortices (BA 10). No region showed significantly greater activity for unaware errors when compared with aware errors. When averaging over aware and unaware errors, no main effect of group was found in any of these error-related regions.

Using the functionally defined clusters from the errorrelated activity map, we conducted an ROI analysis comparing aware and unaware error-related activity separately for each group. Error awareness for control participants was associated with significant differences in four of the awareness ROIs (see Figure 2); greater activity for aware errors was seen in the right insula and left inferior parietal regions when compared with unaware errors, whereas greater deactivation for unaware errors was seen for the right middle occipital and right cerebellar regions. Similar to controls, the cannabis group showed greater activity for aware errors (when compared with unaware errors) in the right insula and left IPL. In contrast to controls, the users also showed aware-unaware differences in the right ACC, right middle frontal and left middle frontal regions when compared with unaware errors, whereas significantly more deactivation during unaware errors was seen for the right putamen and right middle occipital regions. 


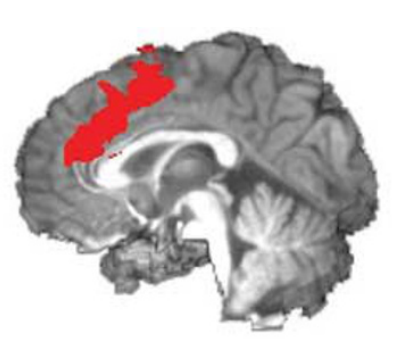

Cannabis Users

Controls
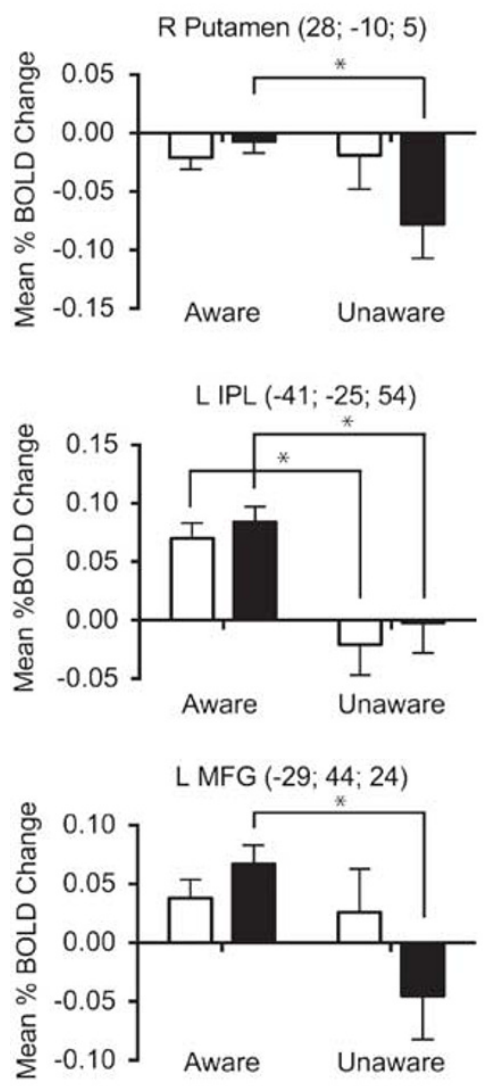

R MOG $(29 ;-92 ; 14)$

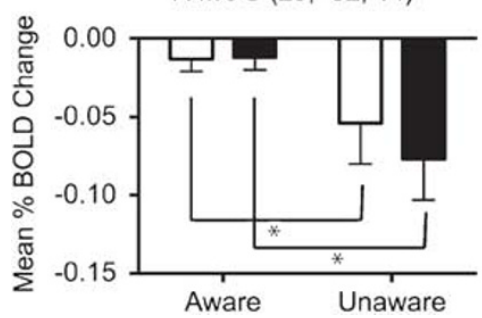

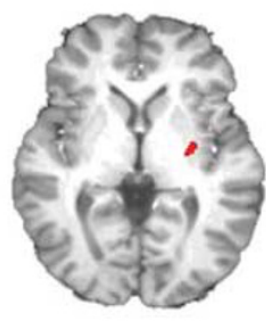
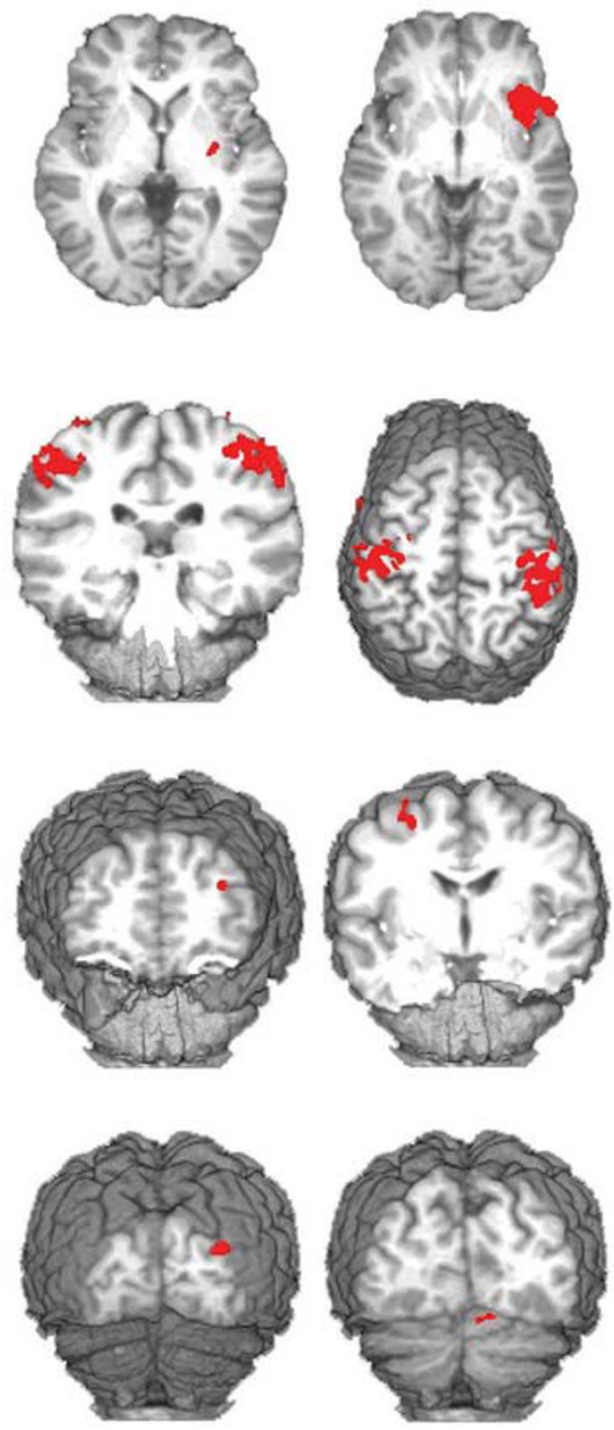

$\operatorname{RACC}(2 ; 21 ; 36)$

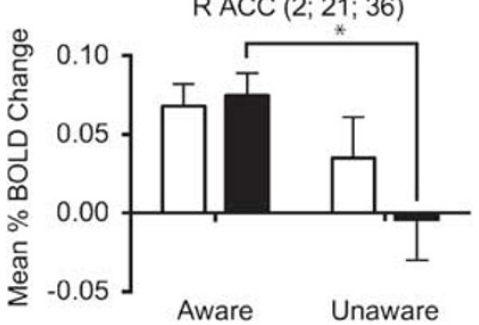

R Insula $(41 ; 15 ;-1)$

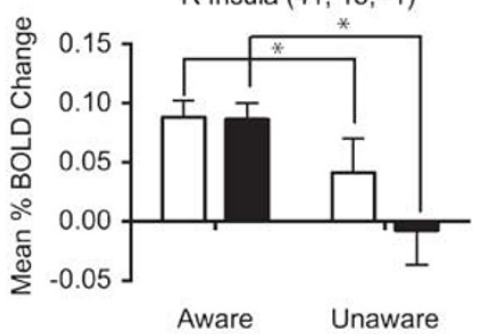

RIPL $(47 ;-34 ; 48)$
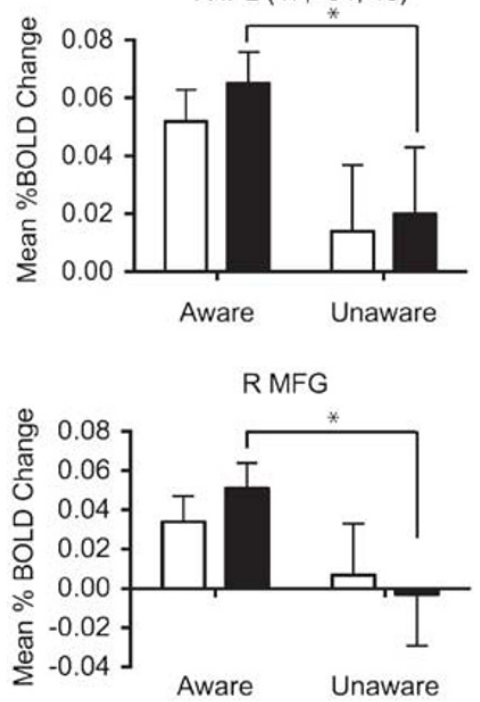

R Cerebellum (13; -80; -21)

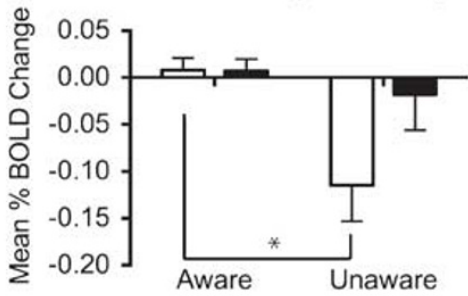

Figure 2 Regions of brain activity differentiating aware from unaware errors. Bar graphs represent mean BOLD \% signal change (relative to baseline) for each group during aware and unaware errors. Error bars represent the standard error of the mean. The MNI co-ordinates for each region are listed in the title and the brain slices shown represent the view at the relevant $x, y$, or $z$-coordinate (eg, coronal slices relate to the $y$-coordinate). Significant within-group comparisons for aware and unaware errors are indicated by the bar and asterisk notations.

Separate between-group comparisons for aware and unaware errors did not show significant group differences in the regions earlier differentiating aware from unaware errors. However, group differences were observed during aware errors in a number of regions that the earlier analysis showed did not differentiate between aware and unaware errors: greater activity was seen for cannabis users in the left putamen and bilaterally in the precuneus, as well as less deactivation in left caudate and left hippocampal regions when compared with control participants.

The relationship between error-related activity and individual differences in error awareness was further examined 
Table 3 Regions of Error-Related BOLD Activity (Combined Across Groups) Differentiating Aware from Unaware Errors

\begin{tabular}{|c|c|c|c|c|}
\hline \multirow[t]{2}{*}{ Brain region } & \multirow[t]{2}{*}{ Volume $(\mu \mathrm{l})$} & \multicolumn{3}{|c|}{ MNI coordinates } \\
\hline & & $x$ & $y$ & $\mathbf{z}$ \\
\hline \multicolumn{5}{|c|}{ Aware errors > unaware errors } \\
\hline $\mathrm{R}$ anterior cingulate & 13377 & 2 & 21 & 36 \\
\hline $\mathrm{R}$ insula & 7663 & 41 & 15 & -1 \\
\hline $\mathrm{L}$ inferior parietal & 7406 & -41 & -25 & 54 \\
\hline $\mathrm{R}$ inferior parietal & 5859 & 47 & -34 & 48 \\
\hline $\mathrm{R}$ middle frontal & 277 & 28 & -8 & 57 \\
\hline R putamen & 206 & 28 & -10 & 5 \\
\hline R middle occipital & 187 & 29 & -92 & 14 \\
\hline R cerebellum (declive) & 177 & 13 & -80 & -21 \\
\hline$L$ middle frontal & 147 & -29 & 44 & 24 \\
\hline
\end{tabular}

using Pearson's correlation coefficients. BOLD activity difference scores were derived for each participant by subtracting unaware from aware error-related activity. Owing to the group differences in behavioral performance the correlation analysis was performed separately in each group. Individual differences in error-awareness levels in the cannabis group significantly correlated with errorrelated activity difference scores in the right ACC $(r=0.50$, $p=0.05)$, and approached significance in the right insula $(r=0.45, p=0.08)$ region. Including inhibition accuracy as a covariate did not alter the significance or strength of relationship between individual differences in error awareness and error-related BOLD activity in the ACC $(r=0.50$, $p<0.05)$ or right insula $(r=0.45, p=0.09)$ for cannabis users. The null effect of this covariate is consistent with the absence of correlation between the behavioral measures of error awareness and inhibition accuracy $(r=0.04)$. Individual differences in error-awareness rates for the control group did not significantly correlate with activity in any of the error-awareness regions. The absence of significant relationships may have been influenced by the limited variability in rates of error awareness for the control group (range $=69 \%$ to $98 \%$ ).

Relationship between cannabis-use behavior and errorrelated BOLD activity. The relationship between errorrelated activity and individual differences in self-reported measures of cannabis use were examined using Pearson's correlation coefficients. BOLD activity scores for each error type were correlated with the indices of use. Aware error activity in the right ACC correlated negatively with reported use in the past month $(r=-0.51, p=0.04)$ and right insula activity correlated negatively with use in the past week $(r=-0.53, p=0.03)$. Unaware error activity in the right insula was negatively correlated with reported use in the past year $(r=-0.56, p=0.03)$. The negative correlations indicate a relationship between higher levels of reported cannabis use and lower BOLD activity for the respective regions.

Self-report measures of craving and withdrawal did not correlate with BOLD activity in any of the error-related ROIs.
Table 4 Regions of Stop-Related BOLD Activity

\begin{tabular}{lcrrr}
\hline Brain region & Volume $(\mu \mathrm{I})$ & \multicolumn{3}{c}{ MNI coordinates } \\
\cline { 3 - 5 } & & \multicolumn{1}{c}{$\boldsymbol{x}$} & $\boldsymbol{y}$ & $\mathbf{z}$ \\
\hline R insula/inferior frontal & 5505 & 41 & 15 & -1 \\
R inferior parietal & 3278 & 49 & $-4 \mid$ & 42 \\
L insula/inferior frontal & 822 & -35 & 15 & -6 \\
R middle cingulate & $59 \mid$ & 2 & -18 & 33 \\
R anterior cingulate & 589 & 5 & 36 & 22 \\
R middle temporal & 379 & 58 & -28 & -1 \\
L inferior parietal & 299 & -39 & -51 & 56 \\
R putamen & 265 & 19 & 6 & -4 \\
L insula & 250 & -29 & 20 & 5 \\
R thalamus (red nucleus) & 234 & 6 & -19 & -2 \\
R middle cingulate/SMA & 168 & 3 & -14 & 49 \\
R middle cingulate & 158 & 1 & -27 & 28 \\
R middle frontal (BA 10) & 153 & 39 & 49 & 18 \\
R middle frontal (BA 9/I0) & 146 & 39 & 40 & 30 \\
\hline
\end{tabular}

Stops. Event-related BOLD activity during Stops indicated 14 clusters of significant activity, including the right prefrontal, parietal, and anterior cingulate regions earlier seen with other versions of the Go/No-go task (see Table 4). A group comparison showed significant differences in three regions, the right IPL, right putamen, and right middle cingulate gyrus. The latter cluster (MNI co-ordinates: $x=3$; $y=-14 ; z=49$ ) is dorsal to the ACC, and falls within the pre-supplementary motor area earlier identified in response inhibition performance (Garavan et al, 2006; Garavan et al, 2002; Mostofsky et al, 2003; Ullsperger and von Cramon, 2001). In all three regions, the pattern of BOLD activity indicated significantly higher levels for the cannabis group when compared with the control group.

\section{DISCUSSION}

Cannabis using participants displayed significantly poorer awareness of errors than a matched control sample. The awareness deficit occurred in the absence of a primary task performance deficit: control and cannabis groups made equivalent numbers of inhibitory control errors from which awareness was assessed. The failure to recognize an error was associated with hypoactive BOLD responses in the cannabis group, in cortical regions including the ACC, right insula, bilateral inferior parietal, and middle frontal regions (when compared with aware error activity). In contrast to control participants and earlier studies examining error awareness (Hester et al, 2005; Klein et al, 2007; Nieuwenhuis et al, 2001), cannabis users did not show significant ACC activity during unaware errors (see Figure 2). One interpretation of these results is that the failure of the ACC (and other regions such as the bilateral middle frontal gyri) to activate for all errors underlies the poor awareness of errors by cannabis users.

The hypoactive error-related ACC response observed in the cannabis group is consistent with similar diminished 
responses in schizophrenia (Alain et al, 2002; Bates et al, 2002; Carter et al, 2001; Kerns et al, 2005; Laurens et al, 2003; Turken et al, 2003), major depression (Steele et al, 2007), ADHD (Liotti et al, 2005; Rubia et al, 2005), and various drug-dependent samples (eg, cocaine (Kaufman et al, 2003), methamphetamine (London et al, 2005), heroin (Forman et al, 2004), and alcohol (Ridderinkhof et al, 2002)). The current study is the first to show that such ACC hypoactivity may be linked to failures of error awareness. Indeed, this data suggest that error-related ACC hypoactivity is specific to those errors of which the participant was not aware. Earlier studies showing error-related ACC hypoactivity had not examined error awareness, or they had provided event-related performance feedback to negate the requirement for error detection. Given the current finding, we speculate that the diminished error-related ACC response observed in psychiatric populations may also be associated with, but not exclusively explained by, deficits in error awareness. For example, we have shown in separate studies of cocaine users that they show deficient error awareness and hypoactive error-related ACC (Hester et al, 2007; Kaufman et al, 2003), and similar studies show the same pattern in schizophrenia (Carter et al, 2001).

BOLD activity in two other cortical regions also seemed to differentiate aware from unaware errors for cannabis users, but not control participants. Cannabis users showed less deactivation in the left middle frontal gyrus (BA 10) and right putamen during unaware errors when compared with their aware errors, whereas control participants showed no difference in these regions. The opposite pattern was observed in the right cerebellum, with control participants showing significantly less activity (deactivation) during unaware errors when compared with aware, whereas the cannabis group showed no difference. Common to both groups was the differentiation of aware from unaware errors by greater activity in the right insula, bilateral IPL, and right middle frontal cortices, as well as significantly less deactivation in the right middle occipital cortex. In support of this group effect, the correlational analyses also found that cannabis group participants with high levels of ACC activity during aware errors (relative to unaware errors) had higher error-awareness rates. A similar, but non-significant $(r=0.45, p=0.08)$, relationship was found between activity in the right insula and error awareness.

Error-related insula activity has earlier been associated with error awareness in healthy controls (Hester et al, 2005; Klein et al, 2007). Klein et al highlighted that such activity was consistent with the hypothesis that insula activity reflects interoceptive awareness. Recent work has also suggested that the insula and interoceptive awareness are critical to drug craving and addiction (Gray and Critchley, 2007; Naqvi et al, 2007; Paulus, 2007), whereby the insula monitors interoceptive 'urges' for rewarding stimuli such as a drug of addiction. These hypotheses suggest insula dysfunction may contribute to impaired interoceptive awareness and heightened experience of drug-related urges, potentially at the expense of other interoceptive signalsfor example, decision-making (Craig, 2009; Paulus, 2007). The relative insensitivity of our cannabis group to detecting errors, which was associated with the absence of right insula activity during unaware errors, would seem to be consistent with this hypothesis. Furthermore, lower levels of insula activity were correlated with higher levels of recent cannabis use, and higher levels of cannabis craving were associated with poor error-awareness rates. Our data did not show a relationship between self-reported craving and insula activity, however, further research might examine if manipulating drug-craving (eg, using drug-related cues) influences insula activity and error awareness during cognitive task performance.

It is also of interest how the diminished capacity for monitoring performance observed here, might contribute to the cognitive control dysfunction that is considered critical to the continuation of drug abuse (Goldstein and Volkow, 2002). Error-related ACC activity is known to prompt subsequent adaptive increases in both cognitive control performance and BOLD activity in regions such as the dorsolateral prefrontal cortex (Garavan et al, 2002), and the diminished responses seen in psychiatric populations have been shown to compromise these adaptive changes (Kerns et al, 2005). The lack of contingency between errors and subsequent task performance in the current study prevents us from examining if the diminished error awareness contributes to subsequent cognitive control dysfunction-for example, the failure to learn from errors that underlies perseverative behavior.

The equivalent cognitive control performance of the cannabis group and their significantly greater activity in right IPL, putamen, and middle cingulate (Pre-SMA) regions during correct inhibition trials (when compared with the control group) is consistent with earlier studies in recently abstinent adolescent (Tapert et al, 2007) and adult (Gruber and Yurgelun-Todd, 2005) cannabis users. All three cortical regions have consistently been associated with successful response inhibition performance in healthy controls (Garavan et al, 2003; Kelly et al, 2004). Studies with cannabis users have shown a consistent pattern of increased activity in prefrontal and parietal regions, with the Tapert et al (Tapert et al, 2007) study (which administered a Go/No-go task) showing increased activity in the pre-SMA and right IPL regions that was also observed here. These results have been interpreted as support for a compensatory mechanism in cannabis users, where maintaining equivalent cognitive control performance requires recruitment of additional cortical regions (than those associated with cognitive control in matched controls), or additional activity from regions common to both groups.

The emergence of a behavioral error-awareness deficit in the cannabis group and the associated BOLD activity differences should also be considered in the context of our sample's demographic and drug use profile. Our sample included young, high-functioning adults who had been using cannabis for the past 8 years from an average onset age of 16 years. Based on previous literature (Pope et al, 2001; Solowij et al, 2002), this sample reported mild-tomoderate levels of recent cannabis use that has typically not been associated with deficits on clinical neuropsychological measures. Consistent with this characterization, the present cannabis group's inhibitory control performance on the Go/ No-go task showed no sign of impairment. The absence of significant group differences in the magnitude of errorrelated BOLD activity, despite the presence of group differences in the regions that were associated with error awareness, might also be consistent with the relatively 
limited duration and frequency of use in our sample. For example, the greater frequency of recent use (past week, month, year) was significantly correlated with lower levels of error-related ACC activity. Given the consistency with which diminished error-related activity has been shown in long-term chronic users of other drugs, the absence of this effect requires follow-up with long-term cannabis users.

The presence of a performance-monitoring deficit and its accompanying BOLD changes in our sample suggest that it may be present in the early stages of drug abuse or may indeed precede use and constitute a risk factor for prolonged cannabis use. This prompts the question of whether such a deficit might contribute to the maintenance of drug use, or represent a deficit that accompanies chronic drug use because of systematic changes in the executive control system that underlies performance monitoring.

\section{ACKNOWLEDGEMENTS}

This research was supported by USPHS grant from the National Institute on Drug Abuse: DA01865-01, Australian Research Council Grant (RH) DP0556602 and Australian National Health and Medical Research Council Career Development Award 519730 (RH). The assistance of Gloria Roberts, Gina Joue, Jennifer Jones, and Ella McCabe are gratefully acknowledged.

\section{DISCLOSURE/CONFLICT OF INTEREST}

Drs Hester, Nestor, and Garavan reported no biomedical financial interests or potential conflict of interest.

\section{REFERENCES}

Alain C, McNeely HE, He Y, Christensen BK, West R (2002). Neurophysiological evidence of error-monitoring deficits in patients with schizophrenia. Cereb Cortex 12: 840-846.

Bates AT, Kiehl KA, Laurens KR, Liddle PF (2002). Error-related negativity and correct response negativity in schizophrenia. Clin Neurophysiol 113: 1454-1463.

Bolla K, Ernst M, Kiehl K, Mouratidis M, Eldreth D, Contoreggi C et al (2004). Prefrontal cortical dysfunction in abstinent cocaine abusers. J Neuropsychiatry Clin Neurosci 16: 456-464.

Carter CS, MacDonald III AW, Ross LL, Stenger VA (2001). Anterior cingulate cortex activity and impaired self-monitoring of performance in patients with schizophrenia: an event-related fMRI study. Am J Psychiatry 158: 1423-1428.

Cox RW (1996). AFNI: software for analysis and visualization of functional magnetic resonance neuroimages. Comput Biomed Res 29: 162-173.

Craig AD (2009). How do you feel - now? The anterior insula and human awareness. Nat Rev Neurosci 10(January): 59-70.

Eldreth DA, Matochik JA, Cadet JL, Bolla KI (2004). Abnormal brain activity in prefrontal brain regions in abstinent marijuana users. Neuroimage 23: 914-920.

Forman SD, Dougherty G, Casey BJ, Siegle G, Braver T, Barch D et al (2004). Opiate addicts lack error-dependent activation of rostral anterior cingulate. Biol Psychiatry 55: 531-537.

Frith CD (1987). The positive and negative symptoms of schizophrenia reflect impairments in the perception and initiation of action. Psychol Med 17: 631-648.

Frith CD, Done DJ (1989). Experiences of alien control in schizophrenia reflect a disorder in the central monitoring of action. Psychol Med 19: 359-363.
Garavan H, Hester R, Fassbender C (2003). The Impact of Individual Differences and Prefrontal Control on Action Monitoring Revealed Through fMRI. In: Ullsperger M, Falkenstein M (eds). Errors, Conflicts, and the Brain. Current Opinions on Performance Monitoring. MPI of Cognition: Leipzig, pp 151-172.

Garavan H, Hester R, Murphy K, Fassbender C, Kelly C (2006). Individual differences in the functional neuroanatomy of inhibitory control. Brain Res 1105: 130-142.

Garavan H, Ross TJ, Murphy K, Roche RA, Stein EA (2002). Dissociable executive functions in the dynamic control of behavior: inhibition, error detection, and correction. Neuroimage 17: 1820-1829.

Goldstein RZ, Volkow ND (2002). Drug addiction and its underlying neurobiological basis: neuroimaging evidence for the involvement of the frontal cortex. Am J Psychiatry 159: $1642-1652$

Gray MA, Critchley HD (2007). Interoceptive basis to craving. Neuron 54: 183-186.

Gruber SA, Yurgelun-Todd DA (2005). Neuroimaging of marijuana smokers during inhibitory processing: a pilot investigation. Brain Res Cogn Brain Res 23: 107-118.

Heishman SJ, Singleton EG, Liguori A (2001). Marijuana Craving Questionnaire: development and initial validation of a selfreport instrument. Addiction 96: 1023-1034.

Hester R, Foxe JJ, Molholm S, Shpaner M, Garavan H (2005). Neural mechanisms involved in error processing: a comparison of errors made with and without awareness. Neuroimage 27: 602-608.

Hester R, Simoes-Franklin C, Garavan H (2007). Post-error behavior in active cocaine users: poor awareness of errors in the presence of intact performance adjustments. Neuropsychopharmacology 32: 1974-1984.

Kalivas PW, Volkow ND (2005). The neural basis of addiction: a pathology of motivation and choice. Am J Psychiatry 162: 1403-1413.

Kaufman JN, Ross TJ, Stein EA, Garavan H (2003). Cingulate hypoactivity in cocaine users during a GO/NOGO task as revealed by event-related fMRI. J Neurosci 23: 7839-7843.

Kelly AM, Hester R, Murphy K, Javitt D, Foxe J, Garavan H (2004). Prefrontal-subcortical dissociations underlying inhibitory control revealed by event-related fMRI. Eur J Neurosci 19: 3105-3112.

Keppel G (1991). Design and Analysis: A Researcher's Handbook. Prentice Hall-Englewood Cliffs: New Jersey.

Kerns JG, Cohen JD, Macdonald III AW, Johnson MK, Stenger VA, Aizenstein $\mathrm{H}$ et al (2005). Decreased conflict- and error-related activity in the anterior cingulate cortex in subjects with schizophrenia. Am J Psychiatry 162: 1833-1839.

Klein TA, Endrass T, Kathmann N, Neumann J, von Cramon DY, Ullsperger M (2007). Neural correlates of error awareness. Neuroimage 34: 1774-1781.

Laurens KR, Ngan ET, Bates AT, Kiehl KA, Liddle PF (2003). Rostral anterior cingulate cortex dysfunction during error processing in schizophrenia. Brain 126(Part 3): 610-622.

Liotti M, Pliszka SR, Perez R, Kothmann D, Woldorff MG (2005). Abnormal brain activity related to performance monitoring and error detection in children with ADHD. Cortex 41(3): $377-388$

London ED, Berman SM, Voytek B, Simon SL, Mandelkern MA, Monterosso J et al (2005). Cerebral metabolic dysfunction and impaired vigilance in recently abstinent methamphetamine abusers. Biol Psychiatry 58: 770-778.

Lysaker PH, Bell MD, Bryson G, Kaplan E (1998). Neurocognitive function and insight in schizophrenia: support for an association with impairments in executive function but not with impairments in global function. Acta Psychiatr Scand 97: 297-301. 
Mostofsky SH, Schafer JG, Abrams MT, Goldberg MC, Flower AA, Boyce A et al (2003). fMRI evidence that the neural basis of response inhibition is task-dependent. Cogn Brain Res 17: 419-430.

Murphy K, Garavan H (2005). Deriving the optimal number of events for an event-related fMRI study based on the spatial extent of activation. Neuroimage 27: 771-777.

Naqvi NH, Rudrauf D, Damasio H, Bechara A (2007). Damage to the insula disrupts addiction to cigarette smoking. Science 315: 531-534.

Nieuwenhuis S, Ridderinkhof KR, Blom J, Band GP, Kok A (2001). Error-related brain potentials are differentially related to awareness of response errors: evidence from an antisaccade task. Psychophysiology 38: 752-760.

O'Connell RG, Dockree PM, Bellgrove MA, Kelly SP, Hester R, Garavan $\mathrm{H}$ et al (2007). The role of cingulate cortex in the detection of errors with and without awareness: a high-density electrical mapping study. Eur J Neurosci 25: 2571-2579.

Paulus MP (2007). Decision-making dysfunctions in psychiatry- altered homeostatic processing? Science 318: 602-606.

Pope Jr HG, Gruber AJ, Hudson JI, Huestis MA, Yurgelun-Todd D (2001). Neuropsychological performance in long-term cannabis users. Arch Gen Psychiatry 58: 909-915.

Ridderinkhof KR, de Vlugt Y, Bramlage A, Spaan M, Elton M, Snel J et al (2002). Alcohol consumption impairs detection of performance errors in mediofrontal cortex. Science 298: 2209-2211.

Ridderinkhof KR, Ullsperger M, Crone EA, Nieuwenhuis S (2004). The role of the medial frontal cortex in cognitive control. Science 306: 443-447.
Rubia K, Smith AB, Brammer MJ, Toone B, Taylor E (2005). Abnormal brain activation during inhibition and error detection in medication-naive adolescents with ADHD. Am J Psychiatry 162: 1067-1075.

Saad Z, Ropella K, DeYoe E, Bandettini P (2003). The spatial extent of the BOLD response. NeuroImage 19: 132-144.

Seltzer B, Vasterling JJ, Yoder JA, Thompson KA (1997). Awareness of deficit in Alzheimer's disease: relation to caregiver burden. Gerontologist 37: 20-24.

Solowij N, Stephens RS, Roffman RA, Babor T, Kadden R, Miller M et al (2002). Cognitive functioning of long-term heavy cannabis users seeking treatment. JAMA 287: 1123-1131.

Steele JD, Kumar P, Ebmeier KP (2007). Blunted response to feedback information in depressive illness. Brain 130(Part 9): 2367-2374.

Tapert SF, Schweinsburg AD, Drummond SP, Paulus MP, Brown SA, Yang TT et al (2007). Functional MRI of inhibitory processing in abstinent adolescent marijuana users. Psychopharmacology (Berl) 194: 173-183.

Turken AU, Vuilleumier P, Mathalon DH, Swick D, Ford JM (2003). Are impairments of action monitoring and executive control true dissociative dysfunctions in patients with schizophrenia? Am J Psychiatry 160: 1881-1883.

Ullsperger M (2006). Performance monitoring in neurological and psychiatric patients. Int J Psychophysiol 59: 59-69.

Ullsperger M, von Cramon DY (2001). Subprocesses of performance monitoring: a dissociation of error processing and response competition revealed by event-related fMRI and ERPs. Neuroimage 14: 1387-1401. 